\title{
A COMMENT ON THE NOVIKOV CONJECTURE
}

\author{
JEROME KAMINKER ${ }^{1}$ AND JOHN G. MILLER
}

\begin{abstract}
It is shown that the Novikov conjecture on the homotopy invariance of characteristic numbers of nonsimply connected manifolds is equivalent to the injectivity of Wall's map from homology of groups to the surgery obstruction groups. These statements are then shown to be equivalent to their analogs for rational equivalences of rational homology manifolds.
\end{abstract}

In [6] Wall observed that the Novikov conjecture on higher signatures follows from the injectivity of a map

$$
l_{*}^{\pi}: H_{*}(B \pi ; \mathbf{Q}) \rightarrow L_{*}(\pi) \otimes \mathbf{Q},
$$

where $L_{*}(\pi)=L_{*}^{h}(\pi)$. He suggested that $l_{*}$ being injective is actually equivalent to the Novikov conjecture. We show in this note that this is the case. Further, using work of Ranicki [5] and Anderson [1], we show that the Novikov conjecture is equivalent to its analog where homotopy equivalences of manifolds are replaced by rational equivalences of rational homology manifolds. We were led to consider these questions while studying an analytic formulation of the Novikov conjecture using the BDF theory of $C^{*}$-algebra extensions [2].

1. The definition of $l_{*}^{\pi}$ and the Novikov conjecture. The Novikov conjecture we take to be the following: Let $M$ and $N$ be closed, oriented topological manifolds with fundamental group $\pi$. Let $\rho_{M}, \rho_{N}$ be the maps into $B \pi$ classifying the universal covers of $M$ and $N$. Suppose there is an orientation preserving homotopy equivalence $h: M \rightarrow N$ making the diagram

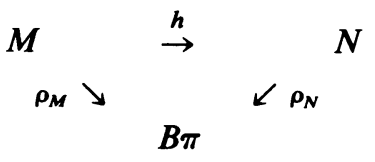

homotopy commute. Let $L(M)$ denote the total Hirzebruch class. Then the Novikov conjecture is that

$$
\rho_{M^{*}}(L(M) \cap[M])=\rho_{N^{*}}(L(N) \cap[N])
$$

in $H_{*}(B \pi ; \mathbf{Q})$. This has the more usual dual formulation that, for all $u \in$ $H^{*}(B \pi ; \mathbf{Q}),\left\langle\rho_{M}^{*} u \cup L(M),[M]\right\rangle=\left\langle\rho_{N}^{*} u \cup L(N),[N]\right\rangle$.

Received by the editors March 17, 1981.

1980 Mathematics Subject Classification. Primary 57R20, 57R67.

${ }^{1}$ Research supported in part by NSF grant MCS-77-21019. 
The map $l_{*}^{\pi}$ is obtained as follows: Let $\Omega_{*}(B \pi)$ denote the oriented topological bordism of $B \pi$. Following Conner and Floyd [3], there is a natural equivalence $\phi$ : $\Omega_{*}(X) \otimes_{\Omega_{*}} \mathbf{Q} \rightarrow H_{*}(X ; \mathbf{Q})$ defined by $\phi([M, f])=f_{*}(L(M) \cap[M])$. The theories are $Z_{4}$-graded and the $\Omega_{*}$-module structure on $\mathbf{Q}$ is provided by the signature.

Define a map $\psi: \Omega_{*}(B \pi) \rightarrow L_{*}(\pi)$ by $\psi([M, f])=f_{*} \theta$, where $\theta$ is the surgery obstruction of a normal map covering

$$
1 \times g: M \times Q^{8} \rightarrow M \times S^{8},
$$

$Q^{8}$ is the Milnor manifold of signature $8, g$ is of degree 1 , and $f_{*}: L_{*}\left(\pi_{1}(M)\right) \rightarrow$ $L_{*}(\pi)$. Then the product formulas for the simply connected surgery obsiruction [4] imply that $\psi$ induces a map

$$
\psi \otimes 1: \Omega_{*}(B \pi) \otimes_{\Omega_{*}} \mathbf{Q} \rightarrow L_{*}(\pi) \otimes \mathbf{Q}
$$

Wall defines $l_{*}^{\pi}$ via the diagram

$$
\begin{array}{ccc}
\Omega_{*}(B \pi) \otimes_{\Omega_{*}} \mathbf{Q} & \stackrel{\psi \otimes 1}{\rightarrow} & L_{*}(\pi) \otimes \mathbf{Q} \\
& & \nearrow l
\end{array}
$$

2. The case of manifolds.

THEOREM 1. The Novikov conjecture holds for the group $\pi$ if and only if $l_{*}^{\pi}$ is injective.

Proof. Suppose given $[M, f],[N, g]$ in $\Omega_{*}(B \pi)$, and an orientation preserving homotopy equivalence $h: M \rightarrow N$ such that $g h \simeq f$. The map $h$ is covered by the bundle map $H: \nu_{M} \rightarrow\left(h^{-1}\right)^{*}\left(\nu_{M}\right)$. By $[6,(17 H)], 8 \theta[h, H]=\psi([N, g])-\psi([M, f])$. Since $h$ is a homotopy equivalence $\psi([M, f])=\psi([N, g])$, and it follows that $l_{*}^{\pi} \phi([M, f])=l_{*}^{\pi} f_{*}(L(M) \cap[M])$ is equal to $l_{*}^{\pi} \phi([N, g])=l_{*}^{\pi} g_{*}(L(N) \cap[N])$. Thus $l_{*}^{\pi}$ injective implies the Novikov conjecture.

Conversely, assume that the Novikov conjecture holds. Suppose that

$$
l_{*}^{\pi} f_{*}(L(M) \cap[M])=0 \text {. }
$$

Then $\psi \otimes([M, f])=0$ in $L_{*}(\pi) \otimes \mathbf{Q}$, so $\psi([M, f])$ has finite order $r$. Let $\left[M^{\prime}, f^{\prime}\right]$ represent $r[M, f]$, so that $\psi\left(\left[M^{\prime}, f^{\prime}\right]\right)=0$. By the argument in [6, Theorem 9.4] there is a bordism of normal maps over $B \pi$ from $M^{\prime} \times Q^{8} \rightarrow M^{\prime} \times S^{8}$ to a normal map $W \rightarrow V$ with $\pi_{1}(V) \cong \pi$. Since the surgery obstruction in $L_{*}(\pi)$ is preserved, this problem is normally cobordant to a homotopy equivalence. Now, using bordism invariance and the homotopy invariance provided by the assumed Novikov conjecture

$$
\left(f p_{1}\right)_{*}\left(L\left(M^{\prime} \times Q^{8}\right) \cap\left[M^{\prime} \times Q^{8}\right]\right)=\left(f p_{1}\right)_{*}\left(L\left(M^{\prime} \times S^{8}\right) \cap\left[M^{\prime} \times S^{8}\right]\right) .
$$

It follows that

$$
f_{*}\left(\left(L\left(M^{\prime}\right) \cap\left[M^{\prime}\right]\right)\right) \sigma\left(Q^{8}\right)=f_{*}\left(\left(L\left(M^{\prime}\right) \cap\left[M^{\prime}\right]\right)\right) \cdot \sigma\left(S^{8}\right) .
$$

Since $\sigma\left(Q^{8}\right)=8$ and $\sigma\left(S^{8}\right)=0$ we obtain $f_{*}\left(L\left(M^{\prime}\right) \cap\left[M^{\prime}\right]\right)=0$. Since $H_{*}(B \pi ; \mathbf{Q})$ is a rational vector space $f_{*}(L(M) \cap[M])=0$ as well. It follows that $l_{*}^{\pi}$ is injective. 
3. Rational homology manifolds. Anderson [1] has established a full analog of Wall's theory for polyhedral rational homology manifolds. The goal of that theory is to find a normal cobordism of a normal map to a rational equivalence. By this is meant an orientation preserving map $f: M \rightarrow N$ with $f_{*}: \pi_{1}(M) \rightarrow \pi_{1}(N)$ and $f_{*}$ : $H_{*}(\tilde{M} ; \mathbf{Q}) \rightarrow H_{*}(\tilde{N} ; \mathbf{Q})$ isomorphisms, where the tildes denote universal covering spaces. The obstruction lies in $L_{*}(Q \pi)$ rather than $L_{*}(\pi)$. The Novikov conjecture for rational homology manifolds is as before, with rational equivalence of rational homology manifolds replacing homotopy equivalence of manifolds. Let $\Omega_{*}^{\mathbf{Q}}(X)$ denote bordism groups of rational homology manifolds. The existence of an equivalence $\phi: \Omega_{*}^{\mathbf{Q}}(X) \otimes_{\Omega_{*}^{\mathbf{Q}}} \mathrm{Q} \rightarrow H_{*}(X ; \mathbf{Q})$ requires no changes from the manifold case, so $\mathbf{Q}_{*}^{\pi}$ may be defined as before.

THEOREM 2. The Novikov conjecture for rational homology manifolds holds for the group $\pi$ if and only if $\mathbf{Q}_{*}^{\pi}$ is injective.

Proof. This follows in exactly the same way as did Theorem 1.

THEOREM 3. The Novikov conjecture for manifolds and for rational homology manifolds are equivalent.

Proof. We have seen that these are equivalent to the injectivity of the naturally related homomorphisms $l_{*}^{\pi}: H_{*}(B \pi ; \mathbf{Q}) \rightarrow L_{*}(\pi) \otimes \mathbf{Q}$ and $\mathbf{Q}_{*}^{\pi}: H_{*}(B \pi ; \mathbf{Q}) \rightarrow$ $L_{*}(\mathbf{Q} \pi) \otimes \mathbf{Q}$. According to Ranicki $[5], L_{*}(\pi) \rightarrow L_{*}(\mathbf{Q} \pi)$ is an isomorphism modulo 8-torsion. The result follows.

\section{REFERENCES}

1. G. A. Anderson, Surgery on $\Lambda$-homology manifolds, Illinois J. Math. 24 (1980), 653-674.

2. R. G. Douglas, $C^{*}$-algebra extensions and $K$-homology, Princeton Univ. Press, Princeton, N.J., 1980.

3. P. E. Conner and E. E. Floyd, Differentiable periodic maps, Springer-Verlag, Berlin and New York, 1964.

4. J. W. Morgan and D. P. Sullivan, The transversality characteristic class and linking cycles in surgery theory, Ann. of Math. (2) 99 (1974), 463-544.

5. A. Ranicki, Localization in quadratic L-theory, Algebraic Topology-Waterloo, Lecture Notes in Math., vol. 741, Springer-Verlag, Berlin and New York, 1979.

6. C. T. C. Wall, Surgery on compact manifolds, Academic Press, New York, 1970.

Department of MATHEmatics, Indinna-PURdue University, Indianapous, Indiana 46205 